\title{
The Active Use of Online Presence, Movies and Gameplay to Improve Classroom Engagement
}

\author{
Sean Costain and Dale Patterson \\ Queensland College of Art, Griffith University, Gold Coast, Australia \\ \{s.costain, d.patterson\}@griffith.edu.au
}

\begin{abstract}
The online world is filled with rich interactive games, spaces, motion pictures and personas. Despite a rapid growth in online education, the tertiary classroom looks quite different to the entertaining online world it exists within. The design of mobile online resources, both official and unofficial, plays a key role in student engagement and learning. From the teachers perspective designing an online presence and in particular engaging online learning resources, is critical to the success of the learning environment. This project looked at the use of popular forms of online materials, including gameplay, movies and social media, and whether the application of entertainment centered tools enhanced the learning environment. The results of the 9 year, 984 participant study indicate that the increased and active use of the entertainment based tools had a significant positive effect in terms of student engagement and a significant positive effect on learning outcomes for international students.
\end{abstract}

Keywords: online learning, student engagement, gameplay, social media.

\section{Introduction}

The online environment, available today to students, offers resources on an enormous scale. Unfortunately the reliability of these resources is less clearly indicated. Prior to the widespread use of the web, the learning and teaching environments used in tertiary education were significantly simpler, both in terms of technology, scale of information and reliability of information. With the addition of mobile online learning resources, online course delivery and social media communications, the tertiary education space is a very different place to that which existed just 25 years ago [1,2]. Today students have immediate access to the pool of rich online resources in a range of interactive forms. Knowledge of facts has become less relevant to these students as they have 24 hour a day, seven day a week, access to information at their fingertips.

The ability to discern valuable information, as compared to the less reliable information, from the mix that exists on the web, becomes a critically important skill for the student $[3,4]$. Aside from pure informational resources, the web also offers a range of richer and more engaging options. These include social media, chat as well as online video and audio tutorials and advice on "how to" complete numerous tasks.

These more interactive and visually richer forms of accessing information appeal to students on many levels, including personal engagement as well as simply being a more entertaining way to obtain the required information $[5,6]$. 
For teaching staff, and those designing learning resources and programs, it is critical to design learning resources that are relevant and effective for the student in this online learning space [1], [7], [8], [9]. To achieve this it would seem logical that the teaching staff would look to using the common forms of online resources that students are seeking out, including short entertaining videos, social media interaction and others, as a means to deliver their resources and knowledge.

This research project implemented a study over a nine year period to identify whether the increased use, by teaching staff, of popular forms of entertainment, in the form of movies, interactive gameplay and social media had an impact on the effectiveness of the learning environments they created.

\section{Online Learning Resources}

Learning resources come in a range of possible forms and formats. For the purpose of this study the focus was in the online delivery of resources based on popular forms of entertainment. This approach included the use, and comparison of the effectiveness of, several types of formats for this content. Key formats included, long and short video and audio clips, interactive gameplay (in both game and social media platforms) as well as the active and passive use of social media.

The short movie format, was a critical element in the trial as these items became the most viewed and used (with more than three times the amount of interaction from participants than any of the other forms). Each of these movies was designed to be much like a short movie trailer only containing key and quite specific pieces of knowledge. These clips were constrained to being no longer than five minutes in length and were designed to be as informal as possible (including showing minor errors and corrections). Several critical additions to each of these resources included the removal of official branding, the inclusion of the person (teaching staff member) in the clips themselves and the publication in personal (non official) locations on the web. The intent of these elements was to help break down the barriers between staff and student and reinforce the links and build rapport between the student and staff.

The same approach was applied to developing the interactive gameplay scenarios and social media interactions. The focus being on short, very specific and helpful "tips" being delivered in a non official format but by a person with whom the student is comfortable, and has trust in the reliability of their information. Interestingly the feedback from the study regarding the interactive gameplay indicated that the teaching staff members willingness to engage in such "play", outside of the content and teaching scope, was the most highly rated item listed in the question asking "Which activity helped you get to know your teacher better?" with 44 percent of participants questioned listing gameplay as the most effective experience. This demonstrated the power of interaction in building relationships, but also shows the ability of a game, though the focus on a separate, and in these cases a shared task, to remove many fears and barriers to communication. The focus on the game play allows communication channels to open (about and in the game) and from that better understanding and ongoing communication was most effectively generated. 
With the web itself having so many unreliable information sources, generating trust is critical to the success of this system. Where the textbooks of the past were a known entity with reliable information, the web contains information that is not edited and in many cases is simply incorrect. This leads to a more information wary student. One who seeks the ready accessibility of the Internet resources, but also questions their true value. In reality, this web based collection of information is what the student often consults first, but due to their uncertainty in the information, they will often cross check with multiple sources. Essentially they perform a simple form of peer review before giving trust to the information obtained.

Developing this level of trust in the teaching staff members "unofficial" content is one of the key challenges. Participant feedback from the study indicated the fact that the staff member was open on a personal level, thus enabling students to make the link between their personal "unofficial" identity and their true real world identity made them more confident in the truth and personal reliability of the teaching staff members virtual persona. With confidence transferred to the reliability of the tips and tricks provided through the short videos of that persona. In simple terms there appears, based on the studies findings, value in truth in virtual personas and also value in more personal detail and engagement.

\section{Online Teaching Presence and Persona}

The learning environment populated with students today is a rich and highly technology based world. For many teaching staff this in itself is challenging. In many cases students are more comfortable with the technology and communication systems than the staff themselves $[7,8]$. The question of how important, in the modern era, an online presence is for an effective teacher is challenging. At its core, teaching involves assisting the student to obtain the knowledge and skills required to understand and successfully deal with challenges. For the modern teacher this means being there to assist with personal face-to-face learning, but also being there to assist with independent learning through online presence, interactive communication and the delivery of engaging learning resources. For the teacher, this makes their online presence a critical element of their professional persona. In the world of online study, a teacher's online presence is often more heavily accessed and utilized than the interpersonal face-to-face engagements. Managing this virtual professional teaching presence, in its many forms and including the use of entertainment techniques to engage and inspire the students, is a skill that has become a valuable skill to all teachers involved in the fast growing online education environment.

The 24/7 nature of online environments makes it almost impossible for any member of teaching staff to be available at all times to students. One of the key techniques pursued in this research was the use of personal entertainment oriented media as a means to capture key elements of knowledge and present them in an engaging and personal online form to students.

One of the key issues with online delivery of materials is the sense of detachment that students feel $[9,10]$. This research experimented with differing levels of "person" 
in the online personas of teaching staff. The objective was to understand what level of personal link is beneficial in building effective forms of communication. To understand and measure the importance of different features of the staff members online presence, the study developed three levels of online teaching resources or presence.

The first level, the control group, contained staff who had no online presence and delivered learning materials in a classic face-to-face teaching manner. Students knew them as a teacher in class but had no additional "personal" information.

The second level, passive virtual presence, contained staff who had an online presence, delivered materials online, but did not actively engage in the online community or use specific targeted personal short videos, interactive games or active use of social media. Students knew them as a teacher in class and also had a limited amount of additional "personal" information through their professional media profiles.

The third level, the active virtual presence, contained staff that had an online presence, delivered materials online and actively engaged in the online community including the use of specific targeted personal short videos and interactive games scenarios. They also pursued active use of social media to communicate with students. Students knew them as a teacher in class and also had a significant amount of additional "personal" information and contact through their detailed personal social media profiles.

\subsection{Mixing Online Teaching Presence and Learning Resources}

Most professional personas link closely to the topic of the professional work being carried out. In these professional scenarios the separation between the individual's persona and the content of the work can often be blurred. For virtual teaching presence this soft boundary between content and person provides an interesting platform for delivering learning materials. Initially the content builds confidence in the online identity, and draws students to it. Over time the identity becomes trusted and the identity itself can actively drive students to resources.

This research project sought to understand the role of the human element or "person" in the virtual teaching presence. Specifically to experiment with different levels of "person" relative to "content" in the virtual personas and to critically review the impact, positive or negative, that these changes caused. The goal being to identify mechanisms through which improvements could be made to a virtual teaching persona's ability to function as an effective teaching tool.

\section{$4 \quad$ Experimentation and Analysis}

To gain a better understanding of the type of resources that were most effective, an experimental study was carried out using variations on teaching presence (these differing levels incorporate different types of learning resources used). The key purpose of the observational study was to identify whether the entertainment oriented 
learning resources (movies, games and social media as included in the active virtual presence) had an impact on the learning outcomes or engagement of students when compared to the control and passive presence groups.

The experimental work involved a randomized control trial comparing the different types of teaching presence. The trial involved four teaching staff and 984 students across a period of nine years. The participants were all students involved in tertiary level classes, and were engaged in the study for six-month periods. Participants ranged in age from 17 to 54 years. They came from a range of cultural backgrounds and were taught in the English language (a second language for many of them).

The trial began with each participant being surveyed and then randomly allocated to one of the three levels of teaching presence (control, passive virtual or active virtual). Each participant then undertook their course/project, using the method as appropriate for their group. Interacting with staff, fellow participants and course materials, submitting appropriate assessment items as required. The topic/course content and assessment were consistent for all participants, thus keeping only the delivery method as the altered factor. During the trial they were also surveyed to gain insight into their feedback on how satisfied and engaged they were with the course and its delivery method. The final data element that was collected looked at how the students engaged with the online resources by recording the methods and times at which they accessed the online resources, providing information on which resources and personas were more or less effective in generating engagement and in driving use of the resources.

At the conclusion of the course an assessment was made of the students learning/creative outcomes in the form of examinations and project based work. These assessments were used to measure the effectiveness of the teaching method in terms of learning outcomes. Participant feedback was also collected as a means to measure participant satisfaction and engagement. From this data comparisons between the methods of teaching, with differing types of virtual personas, were developed.

\subsection{Findings from the Study}

The results from the study show that the use of an active virtual presence and in particular the level to which entertainment based techniques were applied, had positive results in terms of the participants experience when compared to the control group. Three key areas of interest were measured, those being learning outcomes, satisfaction and engagement. The active virtual presence group (which included the short movies, game play and social interaction) was significantly more successful in terms of engagement and satisfaction, and achieved positive improvements in learning outcomes for specific groups within the study.

In the key area of learning outcomes, the differing virtual presence did not cause a statistically significant difference across the full group. Overall the learning outcomes amongst students in the control group, averaging $64.7 \%$, were similar to those amongst both the active, averaging $71.4 \%$, and passive virtual presence groups, averaging $67.6 \%$. When reviewed as $95 \%$ confidence intervals for the range of 
participants, these value ranges crossed indicating that there was no statistically significant difference between the learning outcomes from differing "presence" styles. This indicates that the use of entertainment focused learning resources did not cause any significant change to the learning outcomes of the students.

Although there was no statistically significant difference across the full group, there was a difference in learning outcomes for one specific sub group, participants with English as a second language. For participants with language/culture issues their results showed improvement going from $39 \%$ in control groups, to $43 \%$ in passive virtual presence groups, and most significantly up to $61 \%$ in active online presence groups. This improved ability to engage and transfer knowledge to these particular groups, through the use of online entertainment based methods was unexpected and offers potential. With international students playing such a large role in educational classes, these results were an important finding from this study.

In terms of engagement, two key measures were used. Firstly the quantitative data obtained from recording how and when participants accessed the resources. Secondly the feedback obtained in surveys. These results indicated that accesses to resources were higher in the active virtual presence group than any other group. In fact the activity itself played a key role in this. The study showed that an activity (engagement with resources), whether by staff or student, triggered a chain of interactive events. On average a single active interaction in the community learning space, led to an average of more than seven responses and interactions with information. Hence active engagement with the community led to an increase in the use of learning resources as well as increased communication amongst participants.

True identity also played a role, with the personas that incorporated the teachers identity (in a non threatening "unofficial" form) actively into the gameplay and movie content, gaining more engagement and more active response to interactions. Survey responses indicate that this related to a level of knowing the person and feeling a sense of trust and confidence with them. This confidence was critical as it allowed students to feel comfortable enough in the teaching staff, and most importantly the virtual presence of those staff members, to experiment and make mistakes. In simple terms the unofficial elements of the virtual presence gained more interaction, than the official. Participant feedback indicated it was unofficial and more of an open discussion forum which would not have an impact on results or perceptions of their work.

In one class, with 84 students, the social media unofficial group had 76 members join ( $90 \%$ of students in class optionally choosing to join group). During the 24 hours prior to the first assessment item, there were 62 active engagements compared to the average rate of just over one engagement per day. In contrast the number of participants who engaged with the official learning resources in that same 24-hour period was 32, almost half the rate. Clearly showing a preference for the unofficial social media group over the official online learning group. This was interesting as the resources represent contact with the same person, yet the format (of short more personal and unofficial movies) made them more accessible and desirable to participants. 
This finding was reflected not only in regards to assessment and feedback but also in the key content items. Presentation of short video clips through the unofficial mechanism generated more than twice the amount of views and more than four times the amount of participant interactions than any other type of content item.

The other key area of analysis related to levels of participant satisfaction. In this regard, the control group showed higher levels of community satisfaction than the passive virtual presence group. Although they were learning and interacting, they didn't feel as engaged with the passive virtual presence. The active presence group however was significantly higher, than both the control and passive groups, in terms of satisfaction ratings. This once again indicates that the short videos and interactive games created a higher level of satisfaction amongst the participants.

The participant feedback was for the same course content, assessments, with the same member of teaching staff, with the only difference being a differing set of learning resources (more entertainment based in active group). The results highlight the fact that students in active groups found the online community, mixed with targeted short video, game play and social media interactions more engaging. As a result they were statistically significantly more satisfied than their counterparts in other groups.

\section{Discussion and Conclusions}

The results from this and other studies show that an effective virtual teaching presence can achieve more than just a presentation of the resources and a representation of the member of teaching staff $[4,8,10]$. With clever design, particularly through the use of entertainment based content design and delivery, the virtual presence can provide both an identity for the teaching staff member, and also, and perhaps most importantly, provide a mechanism for actively engaging the students in learning activities and delivery of learning resources [11]. To achieve this involves developing mechanisms to build trust, confidence and communication channels. As the participant responses demonstrated, establishing trust was strongly linked to the amount of true individual personal detail that features in the online presence as well as the early engagement of staff and students [5], [12]. Meeting the teacher in person and linking that back to the virtual identity/persona gave an increased level of confidence. The use of virtual presence in this regard also provides a less intimidating environment than the face-to-face classroom. For some groups, including international students, the ability to engage at a distance enables time to formulate responses and deal with the issues caused through language and culture. As a result international students performed much better in the active virtual presence groups.

Overall the results indicate that the communication channels that were most actively used throughout the study were those of an unofficial form in the format of short personal video tips and tricks delivered through social media platforms.

Teaching involves engagement with the student, helping them to see the relevance of the content to their world, and inspiring them to become passionate about the topic. 
The somewhat distant and cold world of the Internet presents a challenging problem in terms of how to most effectively engage with students. The raw learning materials and information may be of the highest quality, but without an engaging mechanism to draw the students to them, they will be unsuccessful as teaching and learning tools. This is evidenced in studies such as those by Kim et al. that state, "media integration and instructors quality teaching were significant predictors of both social presence and learning satisfaction" [12].

Results from this study demonstrate that the active use of learning resources formatted along the lines of entertainment pieces, and delivered through trusted virtual presence that is "unofficial" in form, are more effective in terms of engagement and satisfaction. Specific groups were also more effective in terms of learning outcomes.

\section{References}

1. Allen, E., Seaman, J.: Going the Distance: Online Education in the United States. Sloan Consortium. PO Box 1238, Newburyport, MA 01950 (2011)

2. hKreber, C., Kanuka, H.: The scholarship of teaching and learning and the online classroom. Canadian Journal of University Continuing Education 32(2) (2013)

3. Harasim, L.: Learning theory and online technologies. Routledge, London (2011)

4. Hattie, J.: Visible learning: A synthesis of over 800 meta-analyses relating to achievement. Routledge, London (2013)

5. Junco, R.: The relationship between frequency of Facebook use, participation in Facebook activities, and student engagement. Computers \& Education 58(1), 162-171 (2012)

6. Meyer, K.: The influence of online teaching on Faculty productivity. Innovative Higher Education 37(1), 37-52 (2012)

7. Palloff, R., Pratt, K.: Lessons from the Virtual Classroom: The Realities of Online Teaching. John Wiley \& Sons, New Jersey (2013)

8. Baran, E., Correia, A., Thompson, A.: Transforming online teaching practice: Critical analysis of the literature on the roles and competencies of online teachers. Distance Education 32(3), 421-439 (2011)

9. Patterson, D.: Creativity in the Online Classroom: Findings from a Five Year Randomized Control Trial. In: Bastiaens, T., Marks, G. (eds.) World Conference on E-Learning in Corporate, Government, Healthcare, and Higher Education, vol. 2012(1), pp. 760-766. AACE (2012)

10. Shaikh, Z., Khoja, S.: Role of Teacher in Personal Learning Environments. Digital Education Review 21 (2012)

11. Patterson, D.: Using Interactive 3D Game Play to Make Complex Medical Knowledge More Accessible. Procedia Computer Science 29, 354-363 (2014)

12. Kim, J., Kwon, Y., Cho, D.: Investigating factors that influence social presence and learning outcomes in distance higher education. Computers \& Education 57(2), 1512-1520 (2011) 